\title{
BUCHBESPRECHUNGEN
}

Michael P. Todaro

Economic development in the Third World. An introduction to problems and policies in a global perspective

London, New York: Longman 1977, XXVI, 445 S., £ 9,95

Das Umdenken in der wissenschaftlichen Beschäftigung mit Problemen der Dritten Welt und der Überwindung von Unterentwicklung seit Anfang der 70er Jahre hat sich jetzt auch in einem umfassenden Textbuch niedergeschlagen. Der USamerikanische Entwicklungsökonom Michael P. Todaro, Deputy Director des Center for Policy Studies beim Population Council in New York, vertritt darin engagiert die Sache der Dritten Welt, wie sie etwa vom Third World Forum formuliert wird. Neben seiner langjährigen Erfahrung als Forscher und Hochschullehrer in allen Entwicklungsregionen kommt ihm dabei seine solide Ausbildung in herkömmlicher Ókonomie zugute. So hat der Leser stets den Eindruck, daß der Autor weiß, wovon er spricht, wenn er die Unbrauchbarkeit oder begrenzte Relevanz etwa von neo-klassischen und neo-keynesianischen Modellen der Wachstums-, Beschäftigungs- oder Außenhandelstheorie kritisiert.

Der Auseinandersetzung mit herkömmlichen Ansätzen der Entwicklungsökonomie dient vor allem Teil I des Buches, der zugleich Basisinformationen über Gemeinsamkeiten und Unterschiede zwischen den Entwicklungsländern liefert und die zu behandelnden Probleme umreißt; sie durchzieht aber auch die problem- und politik-orientierten Teile II und III. Dort werden mit Wachstum, Einkommensverteilung und Beschäftigung, Bevölkerungsentwicklung und Migration, ländlicher Entwicklung und Erziehung sowie Außenhandel, Auslandsinvestitionen und internationalen Finanzen die zentralen Problembereiche und Aufgabengebiete vorgestellt, denen heute nationale wie internationale Entwicklungspolitik gegenübersteht. Die Zukunftsaussichten der Dritten Welt wie der Weltwirtschaft überhaupt und die Möglichkeiten zur Überwindung von Unterentwicklung werden noch einmal zusammenfassend in Teil IV diskutiert. Dabei weist der Autor auf die beschränkte Handlungsfähigkeit des Staates hin, dem gleichwohl die Hauptlast zufällt, und erörtert die Forderungen nach einer "neuen internationalen Wirtschaftsordnung“.

Gegenstand des Buches sind ökonomische Probleme und Lösungsansätze. Doch weist der Autor in für einen Ökonomen bemerkenswerter. Weise auf interdisziplinäre Zusammenhänge hin. Er scheint Myrdals Ansicht zu teilen, daß man nicht zwischen ökonomischen und außerökonomischen Faktoren trennen dürfe, sondern allenfalls zwischen relevanten und weniger relevanten. Neben der Verknüpfung der behandelten Einzelprobleme wird immer wieder die Einbettung der Ơkonomie in soziale, politische, kulturelle und ökologische Zusammenhänge hervorgehoben und darauf hingewiesen, daß die einzelnen Entwicklungsländer. Teil eines ungleich strukturierten internationalen Systems sind.

$\mathrm{Da}$ das Buch eigentlich keine ökonomischen Vorkenntnisse verlangt, flüssig und untechnisch geschrieben ist und die notwendigen technischen Informationen verständlich, aber für den Einstieg ausreichend präzise liefert, eignet es sich nicht nur für Studenten der Entwicklungsökonomie als Einführungstext, sondern spricht auch Studenten anderer Disziplinen und den entwicklungspolitisch inter- 
essierten „Laien“ an. Für diese Lesergruppen bietet ein Glossar der wichtigsten technischen Termini zusätzliche Hilfe. Darüber hinaus gibt Todaro auch für Leser mit höherem Informationsstand einen kompetenten Überblick über die aktuelle entwicklungspolitische Diskussion, der zum Nachdenken über die eigene Position einlädt und vielleicht auch an der einen oder anderen Stelle Widerspruch provoziert. Kleine Fehlinformationen wie die über die EG, zu deren Mitgliedern Österreich und die Schweiz (anstatt Belgien und Irland) gezählt werden (S. 422), stellen eine Ausnahme dar. Sie verdient nur als Kuriosum erwähnt zu werden, das auf die periphere Bedeutung europäischer Details aus globaler Perspektive aufmerksam macht. Der wünschenswerten Verbreitung dieses gelungen Buches könnte allenfalls der nicht unbeträchtliche Preis für die gebundene Ausgabe entgegenstehen. Daher ist zu hoffen, daß ihr bald eine erschwingliche Paperback-Ausgabe folgt.

Alfred Schmidt

\section{EBERHARD OTT}

Die Weltorganisation für Meteorologie (WMO), Band 1 der Reihe „Studien aus dem Institut für Internationales Recht an der Universität Kiel“, Verlag Duncker und Humblot, Berlin, 1976, 174 S.

Mit der Veröffentlichung der vorliegenden Dissertation eröffnet das Institut für Internationales Recht in Kiel eine neue Reihe von Studien. Von den Sonderorganisationen der Vereinten Nationen wird in dieser Arbeit die WMO analysiert, die sich mit der internationalen Zusammenarbeit auf dem Gebiete der Meteorologie befaßt. Wenn der Verfasser eingangs hierzu bemerkt, daß diese Tätigkeit weit weniger von politischen Gegensätzen beeinflußt werde als beispielsweise das Wirken der Hauptorgane der Vereinten Nationen, so gibt es doch heute keine UNOOrganisation, die nicht irgendwie von den jeweiligen politischen Strömungen erfaßt würde. Auch in diesem Falle sind die Bemühungen, sich möglichst auf fachliche Fragen zu beschränken und auftretende politische Probleme der Hauptorganisation zu überlassen, nicht immer erfolgreich gewesen. Auf der anderen Seite hat die mehr als 100 Jahre zurückreichende fachliche Zusammenarbeit bemerkenswerte positive Ergebnisse gezeitigt. Dabei haben sich aus der Erfahrung heraus gewisse Eigenheiten entwickelt, die die Weltorganisation für Meteorologie von anderen Sonderorganisationen der Vereinten Nationen unterscheiden.

Um dies verständlich zu machen, gibt der Verfasser zunächst einen kurzen Rückblick auf die geschichtliche Entwicklung. Aus dem weltweiten Charakter von Wetter und Klima ergab sich schon frühzeitig die Notwendigkeit, einheitliche meteorologische Informationen nicht nur laufend zwischen den verschiedenen Ländern auszutauschen, sondern auch die Weltmeere und die Polargebiete mit einzubeziehen. Die Erfordernisse der internationalen Schiffahrt und später des Luftverkehrs verliehen diesen Bemühungen eine zunehmende praktische Bedeutung. Natürlich können die fachlichen Aspekte in einem Buch, welches vorwiegend einer völkerrechtlichen Analyse gewidmet ist, nur kurz behandelt werden; der Verfasser gibt jedoch genügend allgemeinverständliche Auskünfte hierüber, um auch dem Nichtfachmann die eingetretene Entwicklung verständlich zu machen.

Bereits 1873 erfolgte die Gründung einer „Internationalen Meteorologischen Organisation“ (IMO), aus der dann nach dem Zweiten Weltkrieg die heutige WMO 